\title{
Liquid Chromatography with Electrospray Ionization and Tandem Mass Spectrometry Applied in the Quantitative Analysis of Chitin-Derived Glucosamine for a Rapid Estimation of Fungal Biomass in Soil
}

\author{
Madelen A. Olofsson and Dan Bylund \\ Department of Natural Sciences, Mid Sweden University, 85170 Sundsvall, Sweden \\ Correspondence should be addressed to Madelen A. Olofsson; madelen.olofsson@miun.se
}

Received 2 September 2015; Accepted 12 January 2016

Academic Editor: Frantisek Foret

Copyright (C) 2016 M. A. Olofsson and D. Bylund. This is an open access article distributed under the Creative Commons Attribution License, which permits unrestricted use, distribution, and reproduction in any medium, provided the original work is properly cited.

\begin{abstract}
This method employs liquid chromatography-tandem mass spectrometry to rapidly quantify chitin-derived glucosamine for estimating fungal biomass. Analyte retention was achieved using hydrophilic interaction liquid chromatography, with a zwitterionic stationary phase (ZIC-HILIC), and isocratic elution using 60\% $5 \mathrm{mM}$ ammonium formate buffer (pH 3.0 ) and $40 \%$ ACN. Inclusion of muramic acid and its chromatographic separation from glucosamine enabled calculation of the bacterial contribution to the latter. Galactosamine, an isobaric isomer to glucosamine, found in significant amounts in soil samples, was also investigated. The two isomers form the same precursor and product ions and could not be chromatographically separated using this rapid method. Instead, glucosamine and galactosamine were distinguished mathematically, using the linear relationships describing the differences in product ion intensities for the two analytes. The $m / z$ transitions of $180 \rightarrow 72$ and $180 \rightarrow 84$ were applied for the detection of glucosamine and galactosamine and that of $252 \rightarrow 126$ for muramic acid. Limits of detection were in the nanomolar range for all included analytes. The total analysis time was $6 \mathrm{~min}$, providing a high sample throughput method.
\end{abstract}

\section{Introduction}

Estimation of fungal biomass is useful in the study of nutrient and energy flow relationships in soil and when evaluating fungal infection in food and plant material. Various approaches are available to achieve these ends [1]. Direct quantification through histological analysis [2] is one approach, although its tediousness and tendency to deliver biased values are known disadvantages [3]. Indirect quantification using biochemical markers, on the other hand, can deliver more standardized results. Ergosterol, a sterol found almost exclusively in fungal membranes, can be extracted with $\mathrm{MeOH}$ or $\mathrm{EtOH}$ and quantified using liquid chromatography-ultraviolet (LC-UV) detection $[4,5]$. As ergosterol is believed to be relatively unstable and subject to degradation after fungal death, its quantification is generally assumed to estimate the biomass of metabolically active fungi $[4,6]$. Quantification of phospholipid-derived fatty acids
(PLFAs) using gas chromatography is also an alternative [7]. Phospholipids constitute the main structural elements in the cellular membranes of all living organisms with the exception of archaea, and as PLFAs vary structurally with source they provide more or less selective biomarkers for different species of fungi as well as bacteria [8].

Chitin, a naturally occurring polymer of $\mathrm{N}$-acetyl-D-glucosamine, is the structural building block of fungal cell walls. As invertebrate exoskeletons also contain this polymer, microarthropods can contribute to chitin content in soil, albeit in conceivably minute amounts as arthropod soil biomass is the minimal compared with that of soil microorganisms [9]. Chitin is considered more resistant to degradation than ergosterol and phospholipids and is believed to have a recalcitrant portion of 10 to $15 \%$ of the original biomass [10]. Amino sugar-containing polymers are considered to contribute to 5 to $12 \%$ of soil organic $\mathrm{N}$ [11]. 
Through acid hydrolysis chitin can be degraded into the amino sugar glucosamine $(\mathrm{GlcN})[12,13]$, which in turn can be quantified to estimate total fungal biomass. An alternative is to treat chitin with an alkaline solution to form chitosan, an $N$-deacetylated and partly depolymerized product [14]. Another source of GlcN in soil is peptidoglycan, from bacterial cell walls. Peptidoglycan also contains muramic acid (MA), which is exclusively found in bacteria [15] normally at a 1:1 ratio with GlcN [16], though this may vary in some bacterial species [17]. GlcN is also found to some extent in higher plants, typically as glycoproteins in seeds and in fungal and bacteria-produced antibiotics [15].

Various colorimetric assays have been used in the quantification of GlcN and chitosan. Elson and coworkers [18, 19] used $p$-dimethylaminobenzaldehyde (Ehrlich reagent) to form a red-colored product with both GlcN and chitosan. Later, Tsuji and coworkers [20, 21] developed a procedure, also used by Ride and Drysdale [14], where deaminated $N$-acetylglucosamine was treated with 3-methyl-2benzothiazolone hydrazone hydrochloride (MBTH) and ferric chloride to yield an intense blue color which could be measured spectrophotometrically.

Many amino sugars are associated with microorganisms, though GlcN, galactosamine (GalN), mannosamine (ManN), and MA are the ones most commonly found in soil [22]. Chromatographic separation of amino sugars can be performed in gas or liquid phases. Separation with gas chromatography (GC) is possible following analyte derivatization and subsequent flame ionization detection $[23,24]$. Reversedphase (RP) LC-fluorescence detection is also applicable for the quantification of GlcN and other amino sugars. In such applications, the analytes are derivatized with a fluorescent reagent, for example, 9-fluorenylmethyl chloroformate (FMOC-Cl) or $o$-phthalaldehyde (OPA), either manually [12, 25] or automatically using precolumn derivatization [26, 27]. Derivatization not only enables florescence detection, but also improves retention on the otherwise excessively hydrophobic stationary phases of RP columns. An alternative to RP separation is anion-exchange chromatography with pulsed amperometric detection [28]. Advanced analysis of fungal turnover, measuring the ratio of ${ }^{13} \mathrm{C}$ to ${ }^{12} \mathrm{C}$ for amino sugars, is possible via liquid chromatography-isotope ratio mass spectrometry $[29,30]$ or GC-combustion-isotope ratio mass spectrometry [31]. Quantitative analysis of underivatized GlcN in blood plasma and synovial fluid has lately been realized in the pharmaceutical field via HILIC separation and mass spectrometry (MS) detection [32-34].

This project aimed to develop a simple and rapid method for the estimation of fungal biomass by quantification of underivatized, chitin-derived GlcN in soil, applying LC separation with electrospray ionization and tandem mass spectrometry detection (ESI-MS/MS). MA was included in the analytical method to enable calculation of the bacterial contribution to the total GlcN concentration. In addition, the possibility to distinguish GalN from GlcN by comparing product ion intensities is presented. Chemical structures of GlcN, GalN, and MA can be seen in Figure 1.
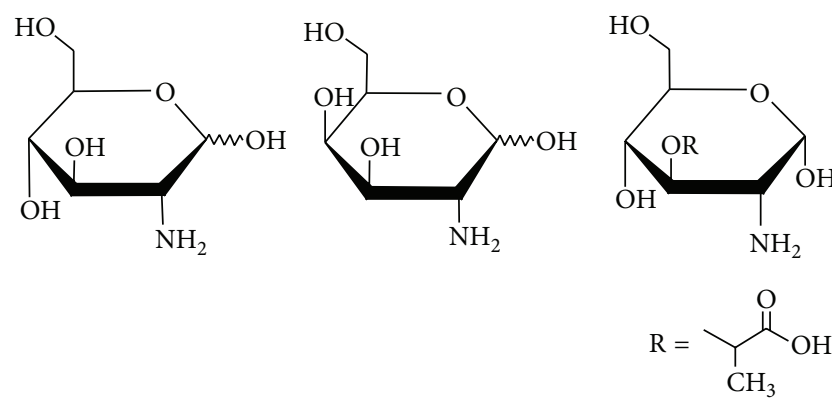

Figure 1: Chemical structures of (from left to right) glucosamine, galactosamine, and muramic acid.

\section{Material and Method}

2.1. Chemicals and Equipment. $10 \mathrm{mM}$ solutions of D-glucosamine hydrochloride (Alfa Aesar, Karlsruhe, Germany) and D-galactosamine hydrochloride (Sigma-Aldrich, St. Louise, USA) were prepared in ultrapure water (MilliQ, Millipore, Bedford, MA). Muramic acid (Sigma-Aldrich, St. Louise, USA) standard solution was prepared by dissolving $5 \mathrm{mg}$ in $10 \mathrm{~mL}$ ultrapure water resulting in an approximate analyte concentration of $2 \mathrm{mM}$. In addition, a $10 \mathrm{mM}$ solution of D-mannosamine hydrochloride (Sigma-Aldrich, St. Louise, USA) was prepared. The standard solutions were stored at $-20^{\circ} \mathrm{C}$ until diluted into working standards. Formic acid for mass spectrometry (Sigma-Aldrich, St. Louis, MO), ammonia (Merck, Darmstadt, Germany), and ACN (for HPLC) from VWR were used in eluents.

The instrumental setup included the following: a Shimadzu LC-10AD isocratic pump (Kyoto, Japan), a mobile phase degassing unit (Uniflows, Tokyo, Japan), an Agilent 1100 autoinjector with appurtenant thermostat (Santa Clara, CA) where the samples were held at $4^{\circ} \mathrm{C}$ prior injection, a ZIC-HILIC analytical column $(150 \times 2.1 \mathrm{~mm}, 5 \mu \mathrm{m})$ from SeQuant (Umeå, Sweden), and an API3000 mass spectrometer (AB Sciex, Concord, Canada).

2.2. LC-ESI-MS/MS Analysis. To achieve sufficient retention and acceptable peak shape, isocratic elution was investigated by combining $5 \mathrm{mM}$ ammonium formate buffer ( $\mathrm{pH}$ 3.0) and $\mathrm{ACN}$ at different percentages. Electrospray and mass spectrometry parameters including collision induced dissociation (CID) analyte fragmentation were investigated via direct infusion of reference material dissolved in the determined mobile phase composition, using a syringe pump (Harvard Apparatus, Holliston, MA). Gas flow settings in the ESI interface and collision cell were optimized manually while analyte-specific potential settings and collision energies were optimized from ramping experiments.

2.3. Method Validation. Limit of detection (LOD) was defined as the injected concentration resulting in a peak height three times the baseline noise level and limit of quantification (LOQ) as the injected concentration resulting in a peak height ten times the baseline noise level. LOD and LOQ were examined through repeated injections of reference material. 
Linearity was investigated from concentrations corresponding to individual LOQ values up to $40 \mu \mathrm{M}$. Precision specific to the peak area was determined via repeated injections of 1 and $10 \mu \mathrm{M}$ standard solution and expressed as relative standard deviation (RSD\%). All standard solutions were dissolved in $1: 1 \mathrm{ACN}$ : ultrapure water.

A number of soil samples were treated according to Ekblad and Nasholm [12], with some minor adjustments. In short, dried and grounded soil was treated with $0.2 \mathrm{~N} \mathrm{NaOH}$ at $70^{\circ} \mathrm{C}$ for $17 \mathrm{~h}$, washed four times with ultrapure water, dried, and finally subjected to acid hydrolysis by additions of $6 \mathrm{M} \mathrm{HCl}$ and sample incubation at $70^{\circ} \mathrm{C}$ for $16 \mathrm{~h}$. Prior to analysis, the acid hydrolysate was filtered through Whatman $0.45 \mu \mathrm{m}$ membrane filters (Clifton, $\mathrm{NJ}$ ), evaporated in $\mathrm{N}_{2}$ flow, and finally resolved in 1:1 ACN : ultrapure water. These samples were analyzed in duplicate and in random order to assess method applicability. Withal, reference material was added to sample matrix to investigate analyte recovery. Standard additions were made at two levels, low and high, corresponding to final sample concentrations of 5 and $20 \mu \mathrm{M}$, respectively. Sample matrix without standard addition, but with the equivalent additions of $1: 1 \mathrm{ACN}$ : ultrapure water, was also analyzed. Six replicates of low, high, and zero additions were analyzed.

\section{Results and Discussion}

3.1. Analytical Parameters. This study aimed to find a simple, rapid method for quantifying chitin-derived GlcN without the need for analyte derivatization. Current methods for quantification of amino sugars in soil use derivatization, which necessitates further sample preparation and additional use of chemicals. Automatic derivatization requires specialized autoinjectors, and manual derivatization can introduce human error. Derivatization is not necessary in MS detection as the analytes are detected according to mass to charge ratio $(\mathrm{m} / z)$. MS/MS detection applying multiple reaction monitoring (MRM) mode is also highly selective, which is favorable when analyzing complex sample matrices.

Since underivatized amino sugars are too polar to be retained on RP stationary phases, HILIC was tested for this purpose. HILIC combines hydrophilic stationary phases with typical RP mobile phases, enabling the retention of polar and hydrophilic analytes [35]. Standard HILIC mobile phases are also compatible with MS detection and typically consist of $40-97 \%$ of ACN or other water-miscible organic solvents. The higher the organic solvent percentage, the higher the retention of analytes. For this application, a zwitter-ionic stationary phase was chosen, consisting of positively charged quaternary ammonium and negatively charged sulfonate groups $(1: 1)$ able to retain both positively and negatively charges analytes.

3.2. Amino Sugar Isomers. GlcN, GalN, and ManN are isobaric amino sugars found in soil. They all produce identical precursor ions in terms of $\mathrm{m} / z$ when analyzed with MS. They also give rise to the same product ions when subjected to CID. These are therefore undistinguishable merely using
MS or MS/MS detection. Even though GlcN is the analyte of interest for the indirect estimation of fungal biomass, coquantification with its isomers may lead to inaccurately high values. For this reason, chromatographic separation of the three isobaric amino sugars was initially tested using gradient elution from a high to low ACN percentage. These attempts proved futile, and even the $\alpha$ - and $\beta$-anomers of the amino sugars appeared to separate more readily than the isomers themselves.

The most intense product ion produced by GlcN, GalN, and ManN had an $m / z$ value of 72 , making it a natural choice for quantification purposes. A closer investigation of the fragmentation patterns of GlcN and GalN, however, revealed differences in intensity among the additional fragments (Figure 2). The most striking difference was observed for the fragment with the $m / z$ value of $84 \mathrm{Th}$, which is produced in significantly greater proportion for GlcN than for GalN. Presumably, these differences in fragment intensities are due to the difference in conformation of the hydroxy group at $\mathrm{C} 4$ and consequently the readiness to lose a second $\mathrm{H}_{2} \mathrm{O}$ group (Figure 2(c)). These differences were not observed between GlcN and ManN, where nearly identical product ion spectra were produced. Since the contribution of ManN to the total amino sugar content in soil is reported to be relatively low, up to 50 times less than GlcN in grass land [36], its distinction was not considered to be critical in quantifying chitin-derived $\mathrm{GlcN}$, and it is therefore not considered further.

The difference found in fragment intensity between GlcN and GalN suggested that they could be distinguished mathematically. Initial tests showed that the ratios between the 72 and 84 Th fragments were reproducible at different analyte concentrations. A full factorial design using Minitab 16 (Minitab Inc., State Collage, Pennsylvania) was performed to investigate this further. Combinations of three levels of individual amino sugar concentrations in mobile phase were analyzed in random order and with two replicates. The design can be found in Table 1. GalN was investigated at higher concentrations compared to GlcN, due to its low formation of the 84 Th fragment.

The results from the full factorial design are also reported in Table 1. A regression analysis was performed on the given areas to investigate a potential relationship. The following linear relationships were found:

$$
\begin{aligned}
& A_{72}=k_{1} \times C_{\mathrm{GlcN}}+k_{2} \times C_{\mathrm{GalN}}, \\
& A_{84}=k_{3} \times C_{\mathrm{GlcN}}+k_{4} \times C_{\mathrm{GalN}} .
\end{aligned}
$$

$A_{72}$ and $A_{84}$ represent areas of the 72 and $84 \mathrm{Th}$ product ions, respectively, and $k_{1-4}$ represents the coefficients of slope specific for each combination of product ion and analyte (GlcN and GalN). The multiple linear regression models were significant with high $R^{2}$ values (97.6 and $98.2 \%$ for the 72 and 84 Th fragments, resp.), and no significant lack-of-fit was noted; that is, the models are valid over the entire range of concentrations studied. 


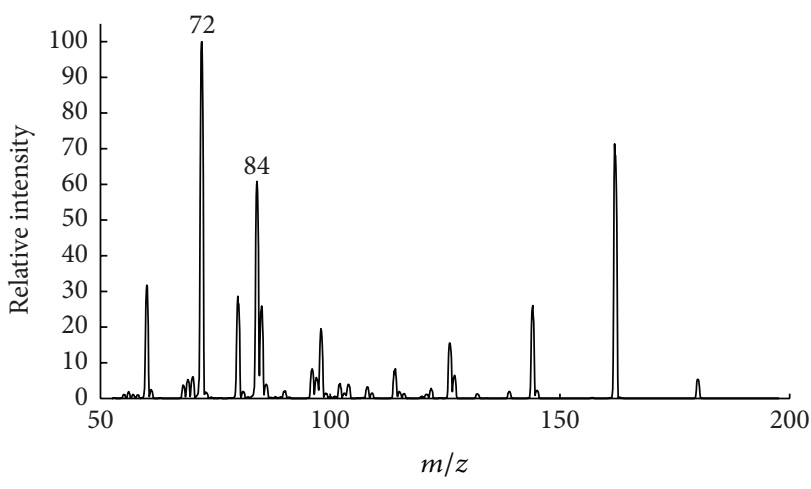

(a)

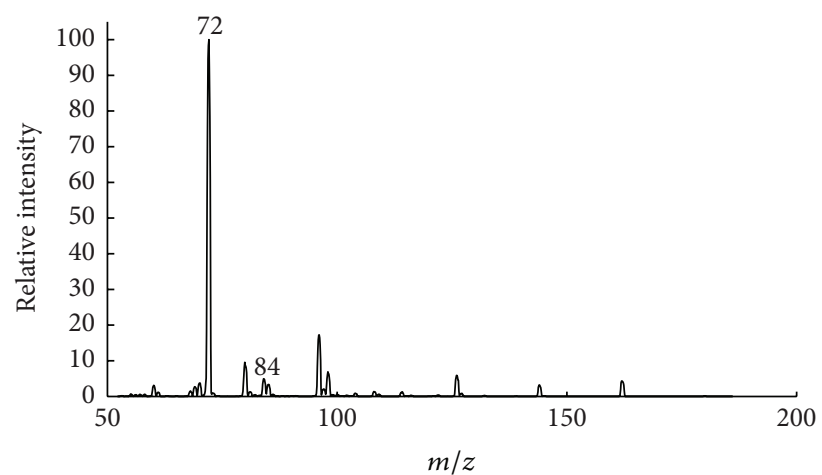

(b)

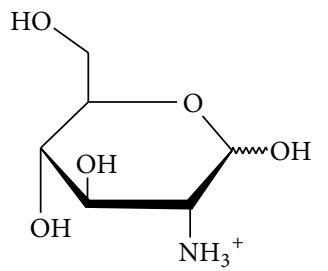

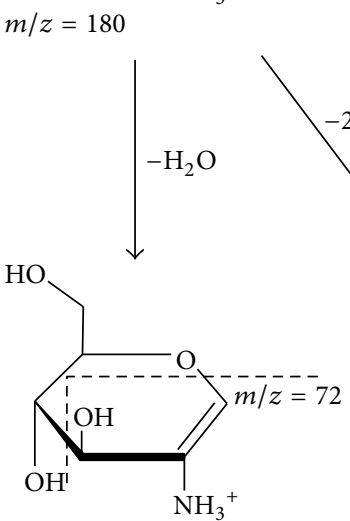

$m / z=162$

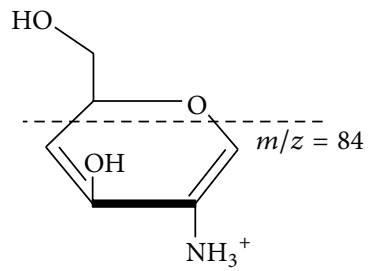

$m / z=144$

(c)

FIGURE 2: Positive ESI-MS/MS product ion spectra of (a) glucosamine and (b) galactosamine, produced through direct infusion of $100 \mu \mathrm{M}$ reference solutions of each analyte with a collision energy of $25 \mathrm{~V}$. Suggested fragmentation of glucosamine (c) presenting the formation of the two $\mathrm{m} / z$ transitions investigated in the method $(180 \rightarrow 72$ and $180 \rightarrow 84)$. Fragmentation of galactosamine is believed to follow the same route but with less formation of the 84 fragment due to the conformation of the C4 hydroxy group.

When solving for $C_{\mathrm{GlcN}}$ and $C_{\mathrm{GalN}}$ using (1) and (2), the following equations were derived:

$$
\begin{aligned}
C_{\mathrm{GlcN}} & =\frac{A_{72}-k_{2} \times C_{\mathrm{GalN}}}{k_{1}}, \\
C_{\mathrm{GalN}} & =\frac{\left(A_{84}-\left(k_{3} \times A_{72}\right) / k_{1}\right)}{\left(k_{4}-\left(k_{2} \times k_{3}\right) / k_{1}\right)} .
\end{aligned}
$$

With (3) and (4), corresponding concentration values for GlcN and GalN can be calculated. This was verified by inserting coefficients of slope from calibration curves determined from standard solutions consisting of reference material in mobile phase, analyzed within the same acquisition batch as the solutions representing the full factorial design. The calculated concentrations are also presented in Table 1 and were found to be on average 10 and $2 \%$ above the true values for GlcN and GalN, respectively.

3.3. Final LC-ESI-MS/MS Method. The sample injection volume was $4 \mu \mathrm{L}$ and the syringe was washed between injections to prevent carry-over effects. Isocratic elution was then achieved using a mobile phase consisting of $60 \% 5 \mathrm{mM}$ ammonium formate buffer ( $\mathrm{pH} 3.0)$ and $40 \% \mathrm{ACN}$, delivered at a flow rate of $0.3 \mathrm{~mL} / \mathrm{min}$. Before entering the ESI interface, the total flow was split using a Valco tee union resulting in a continuous flow of approximately $60 \mu \mathrm{L} / \mathrm{min}$, which is more suitable for ESI. MRM was applied with an analyte $m / z$ transition of $180 \rightarrow 72$ for GlcN, in accordance with Roda et al. [32]. The same optimal $m / z$ transition was applied for GalN. MA was detected at $m / z$ transition $252 \rightarrow 126$, in accordance with Black et al. [37]. In addition, the $\mathrm{m} / z$ transition of 
TABLE 1: In and output values of the full factorial design used to investigate the linear relationship of peak area for the 72 and 84 Th fragments in relation to known concentrations of glucosamine $(\mathrm{GlcN})$ and galactosamine $(\mathrm{GalN})$. Experiments were analyzed in random order.

\begin{tabular}{|c|c|c|c|c|c|c|}
\hline \multirow[b]{2}{*}{ Experiment } & \multicolumn{2}{|c|}{ Conc. $^{1}(\mu \mathrm{M})$} & \multicolumn{2}{|c|}{ Peak area (cps) } & \multicolumn{2}{|c|}{ Calculated conc. $^{2}(\mu \mathrm{M})$} \\
\hline & GlcN & GalN & $180>72$ & $180>84$ & GlcN & GalN \\
\hline 1 & 1 & 5 & $3.6 E 5$ & $3.3 E 4$ & 1 & 5 \\
\hline 2 & 1 & 10 & $5.8 E 5$ & $4.1 E 4$ & 1 & 10 \\
\hline 3 & 1 & 15 & $9.9 E 5$ & $6.1 E 4$ & 1 & 16 \\
\hline 4 & 8 & 5 & $5.3 E 5$ & $1.3 E 5$ & 9 & 5 \\
\hline 5 & 8 & 10 & $8.0 E 5$ & $1.4 E 5$ & 9 & 10 \\
\hline 6 & 8 & 15 & $1.2 E 6$ & $1.7 E 5$ & 9 & 16 \\
\hline 7 & 15 & 5 & $6.9 E 5$ & $2.2 E 5$ & 16 & 5 \\
\hline 8 & 15 & 10 & $9.7 E 5$ & $2.3 E 5$ & 16 & 10 \\
\hline 9 & 15 & 15 & $1.4 E 6$ & $2.8 E 5$ & 16 & 15 \\
\hline 10 & 1 & 5 & $3.3 E 5$ & $2.9 E 4$ & 1 & 5 \\
\hline 11 & 1 & 10 & $6.0 E 5$ & $4.1 E 4$ & 1 & 11 \\
\hline 12 & 1 & 15 & $1.0 E 6$ & $6.1 E 4$ & 1 & 16 \\
\hline 13 & 8 & 5 & $5.9 E 5$ & $1.5 E 5$ & 9 & 5 \\
\hline 14 & 8 & 10 & $9.0 E 5$ & $1.6 E 5$ & 9 & 11 \\
\hline 15 & 8 & 15 & $1.2 E 6$ & $1.7 E 5$ & 8 & 15 \\
\hline 16 & 15 & 5 & $7.9 E 5$ & $2.6 E 5$ & 16 & 5 \\
\hline 17 & 15 & 10 & $9.8 E 5$ & $2.4 E 5$ & 17 & 10 \\
\hline 18 & 15 & 15 & $1.3 E 6$ & $2.5 E 5$ & 16 & 15 \\
\hline
\end{tabular}

${ }^{1}$ Concentrations of GlcN and GalN prepared for each experiment

${ }^{2} \mathrm{GlcN}$ and GalN concentrations calculated using (3) and (4) with slope coefficients $(k)$ derived from coefficients of slope from calibration curves determined from standard solutions analyzed within the same acquisition batch.

TABLE 2: Analyte-specific mass spectrometer parameters applied in the reported method: DP: declustering potential, FP: focusing potential, EP: entrance potential, CE: collision energy, and CXP: cell exit potential.

\begin{tabular}{|c|c|c|c|c|c|c|c|}
\hline Analyte & Precursor ion $(m / z)$ & Product ion $(m / z)$ & $\mathrm{DP}(\mathrm{eV})$ & $\mathrm{FP}(\mathrm{eV})$ & $\mathrm{EP}(\mathrm{eV})$ & $\mathrm{CE}(\mathrm{eV})$ & $\mathrm{CXP}(\mathrm{eV})$ \\
\hline Glucosamine/galactosamine & $180[\mathrm{M}+\mathrm{H}]^{+}$ & 72 & 25 & 100 & 12 & 23 & 13 \\
\hline Glucosamine/galactosamine & $180[\mathrm{M}+\mathrm{H}]^{+}$ & 84 & 25 & 100 & 12 & 21 & 13 \\
\hline Muramic acid & $252[\mathrm{M}+\mathrm{H}]^{+}$ & 126 & 30 & 90 & 9 & 25 & 13 \\
\hline
\end{tabular}

$180 \rightarrow 84$ was included for the mathematical distinction of GlcN and GalN. The amino sugars were analyzed in positive mode applying an ionization voltage of $4500 \mathrm{~V}$. Optimal and analyte-specific declustering, focusing, and entrance potentials are reported in Table 2 . Nebulizer and curtain gas settings were 10 and 8 arbitrary units of $\mathrm{N}_{2}$, respectively. Collision gas was set to 5 arbitrary units of $\mathrm{N}_{2}$ in order to achieve the most favorable fragmentation. Analyte-specific collision energies and collision cell exit potentials are also reported in Table 2. MA eluted at $2.1 \mathrm{~min}$ and GlcN and GalN at $4.5 \mathrm{~min}$ (Figure 3), and the total analysis time was $6 \mathrm{~min}$.

3.4. Method Validation. Method validation was performed on all three analytes and for both the 72 and 84 Th fragments for GlcN and GalN. Linearity was found to be excellent from LOQ values up to $40 \mu \mathrm{M}$. At higher concentrations, the response gradually leveled off. Precision at low and high concentrations lay between 0.6 and $1.8 \%$, and LOD values are between 10 and $500 \mathrm{nM}$. A complete list of figures of merits for GlcN, GalN, and MA is presented in Table 3.
In the recovery trial, recoveries of 95 to $105 \%$ were obtained for MA. Somewhat lower recoveries, in the range of 75 to $85 \%$, were obtained for GlcN and GalN irrespective of the $m / z$ transition $(180>72$ or $180>84)$ investigated. In order to investigate the possibility of coeluting matrix interferences causing this suboptimal recovery, samples were analyzed in full scan mode using both positive and negative electrospray. Possible interferences were discovered, eluting just before GlcN/GalN at $4.2 \mathrm{~min}$, with $\mathrm{m} / z$ values of 64 and 105. These values correspond with the masses of $[\mathrm{ACN}+$ $\mathrm{Na}]^{+}$and $[2 \mathrm{ACN}+\mathrm{Na}]^{+}$. If correctly interpreted, this indicates that a large amount of $\mathrm{Na}^{+}$is present in the sample, presumably as remnants from the $\mathrm{NaOH}$ treatment prior to acid hydrolysis in the sample extraction. It is possible that the repeated washing steps did not adequately remove the access $\mathrm{Na}^{+}$. The addition of $\mathrm{NaOH}$ is believed to remove proteins and amino acids, possible interfering with analysis [12]. This step is, however, often excluded [24, 26, 28-30], which would presumably result in fewer matrix-related problems when applying the analysis method reported here. 
TABLE 3: LC-MS/MS method performance of retention, linearity, sensitivity, precision, limit of detection, and limit of quantification for glucosamine, galactosamine, and muramic acid.

\begin{tabular}{lccccccc}
\hline Analyte & $m / z$ transition & $t_{r}(\mathrm{~min})$ & Linearity $^{\mathrm{a}}$ & Sensitivity $^{\mathrm{b}}$ & Prec. L/H $^{\mathrm{c}}(\%)$ & $\mathrm{LOD}^{\mathrm{d}}(\mu \mathrm{M})$ & $\mathrm{LOQ}^{\mathrm{e}}(\mu \mathrm{M})$ \\
\hline Glucosamine & $180>72$ & $4.41 \pm 0.04$ & 0.9997 & $3.8 E 4 \pm 0.33 E 4$ & $1.3 / 1.0$ & 0.025 & 0.07 \\
Glucosamine & $180>84$ & $4.41 \pm 0.04$ & 0.9997 & $2.1 E 4 \pm 0.17 E 4$ & $0.8 / 0.7$ & 0.05 & 0.2 \\
Galactosamine & $180>72$ & $4.52 \pm 0.03$ & 0.9999 & $8.1 E 4 \pm 0.78 E 4$ & $0.3 / 0.7$ & 0.01 & 0.03 \\
Galactosamine & $180>84$ & $4.52 \pm 0.03$ & 0.9997 & $3.8 E 3 \pm 0.38 E 3$ & $1.8^{*} / 1.5$ & 0.5 & 1.2 \\
Muramic acid & $252>126$ & $2.01 \pm 0.01$ & 0.9994 & $5.1 E 4 \pm 0.20 E 4$ & $1.0 / 0.6$ & 0.01 & 0.03 \\
\hline
\end{tabular}

${ }^{\mathrm{a}}$ Pearson correlation coefficient $(r)$ determined for seven points, ranging from LOQ to $40 \mu \mathrm{M}(n=6)$.

${ }^{\mathrm{b}}$ Regression slope expressed in area units (counts) $/ \mu \mathrm{M}(n=5)$.

${ }^{\mathrm{c}}$ Precision expressed as relative standard deviation (RSD) for repeated injections at $1 \mu \mathrm{M}(\mathrm{L})$ and $10 \mu \mathrm{M}(\mathrm{H})(n=8)$.

${ }^{\mathrm{d}}$ Limit of detection reported as the injected concentration giving a peak height corresponding to three times the baseline noise level.

${ }^{\mathrm{e}}$ Limit of quantification reported as the injected concentration giving a peak height corresponding to ten times the baseline noise level.

${ }^{*}$ Precision at low level was investigated at a concentration of $2 \mu \mathrm{M}$ for the $180>84$ transition of galactosamine, due to its relatively high LOD.

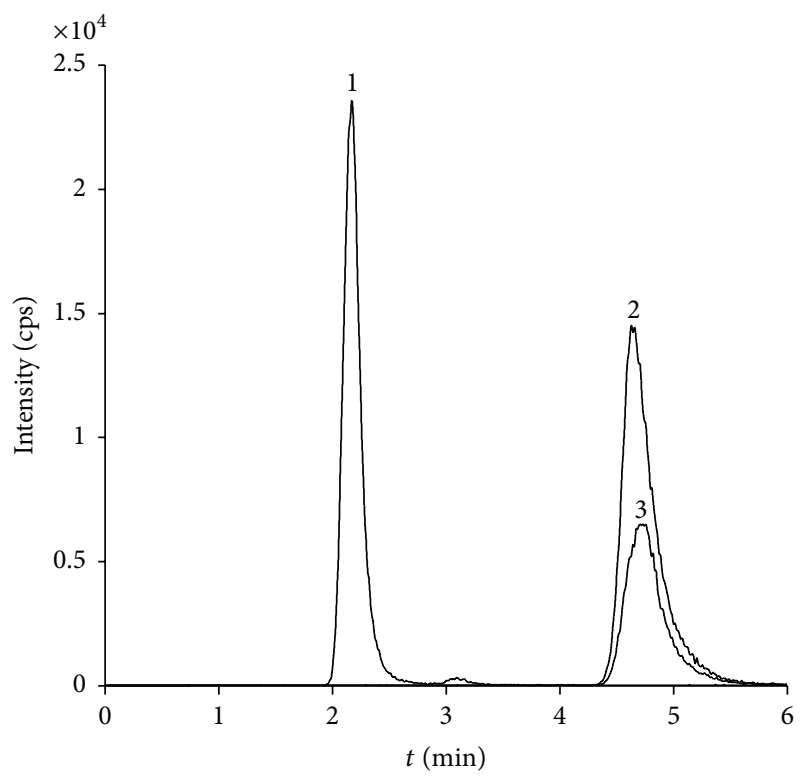

FIGURE 3: MRM chromatogram of a $10 \mu \mathrm{M}$ standard solution of (1) muramic acid and (2) glucosamine $(180>72)$ and $(3)$ glucosamine $(180>84)$ separated on a ZIC-HILIC column $(150 \times 2.1 \mathrm{~mm}, 5 \mu \mathrm{m})$.

3.5. Method Application. Nineteen soil samples, prepared according to the description in Section 2.3, were analyzed with duplicate injections in random order using the presented method. GlcN and GalN concentrations were calculated using (3) and (4) with slope coefficients $(k)$ determined from reference solutions of GlcN and GalN, analyzed within the same acquisition batch. MA was also quantified for the samples, and derived concentrations for the three analytes were compared in terms of relative standard deviation for the duplicate samples. The pooled relative standard deviations for all nineteen samples were 4, 3, and 5\% for MA, GlcN, and GalN, respectively. Figure 4 represents one of the extracted soil samples. Calculated concentrations for GlcN and GalN were 15.3 and $1.4 \mu \mathrm{M}$, respectively. MA concentration was $0.5 \mu \mathrm{M}$, which can be subtracted from the calculated GlcN concentration to compensate for bacterial contribution.

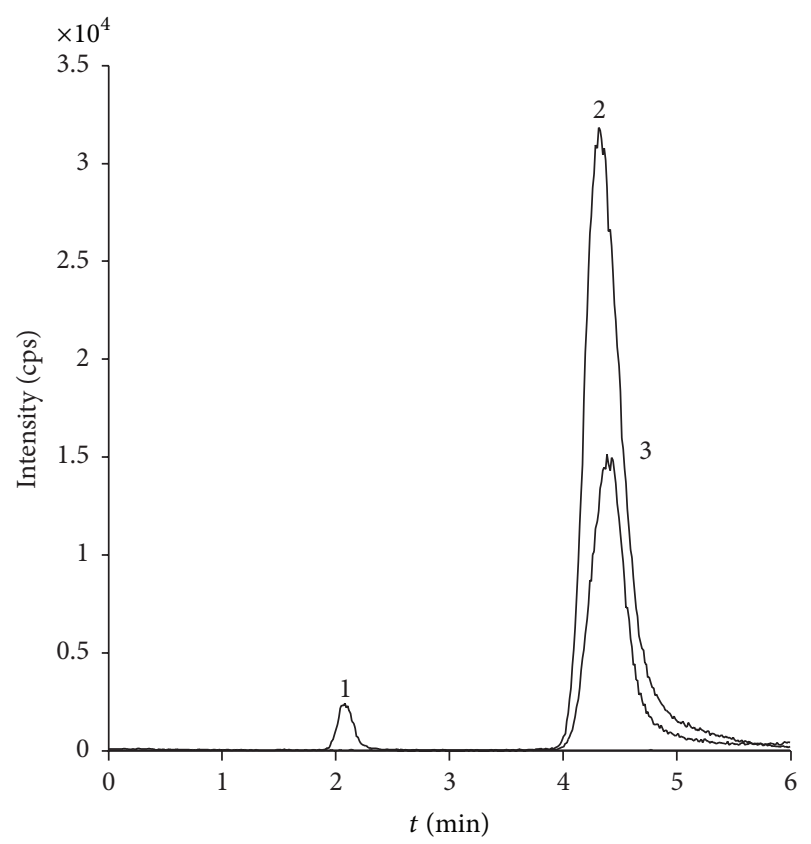

FIGURE 4: MRM chromatogram of an extracted soil sample with peaks representing $m / z$ transitions (1) $252 \rightarrow 126$, (2) $180 \rightarrow 72$, and (3) $180 \rightarrow 84$. Peak areas correspond to $0.5 \mu \mathrm{M}$ of muramic acid and 15.3 and $1.4 \mu \mathrm{M}$ of glucosamine and galactosamine, respectively.

\section{Conclusions}

This paper presents a simple, rapid approach for the estimation of fungal biomass via quantification of chitin-derived GlcN, using LC-ESI-MS/MS with isocratic elution. We believe that this method can beneficially complement the more complex and time-consuming methods that employ analyte derivatization to achieve acceptable separation of the four amino sugars discussed in this paper. By implementing MA in the analytical method, it is possible to deduce the bacterial contribution to the GlcN concentration. MA and GlcN are separated applying HILIC separation, and derivatization is not necessary to achieve chromatographic retention and 
enable detection. Additionally, a linear relationship was found for GlcN and its isomer GalN by investigating intensity differences for two $m / z$ transitions $(180 \rightarrow 72$ and $180 \rightarrow 84$ ), making it possible to mathematically calculate their respective contributions. The total analysis time is $6 \mathrm{~min}$, allowing analysis of high sample throughput to be performed.

\section{Conflict of Interests}

The authors declare that there is no conflict of interests regarding the publication of this paper.

\section{Acknowledgments}

This work was funded by the European Regional Development Fund of EU, Sveaskog, and the County Administrative Board of Västernorrland.

\section{References}

[1] R. G. Joergensen and F. Wichern, "Quantitative assessment of the fungal contribution to microbial tissue in soil," Soil Biology and Biochemistry, vol. 40, no. 12, pp. 2977-2991, 2008.

[2] M. Bölter, J. Bloem, K. Meiners, and R. Möller, "Enumeration and biovolume determination of microbial cells-a methodological review and recommendations for applications in ecological research," Biology and Fertility of Soils, vol. 36, no. 4, pp. 249-259, 2002.

[3] M. Giovannetti and B. Mosse, "An evaluation of techniques for measuring vesicular arbuscular mycorrhizal infection in roots," New Phytologist, vol. 84, no. 3, pp. 489-500, 1980.

[4] B. B. Salmanowicz and J. E. Nylund, "High performance liquid chromatography determination of ergosterolas a measure of ectomycorrhiza infection in Scots pine," European Journal of Forest Pathology, vol. 18, no. 5, pp. 291-298, 1988.

[5] F. Martin, C. Delaruelle, and J. L. Hilbert, "An improved ergosterol assay to estimate fungal biomass in ectomycorrhizas," Mycological Research, vol. 94, no. 8, pp. 1059-1064, 1990.

[6] L. M. Seitz, H. E. Mohr, R. Burroughs, and D. B. Sauer, "Ergosterol as an indicator of fungal invasion in grains," Cereal Chemistry, vol. 54, no. 6, pp. 1207-1217, 1977.

[7] Å. Frostegård, E. Bååth, and A. Tunlio, "Shifts in the structure of soil microbial communities in limed forests as revealed by phospholipid fatty acid analysis," Soil Biology and Biochemistry, vol. 25, no. 6, pp. 723-730, 1993.

[8] L. Zelles, "Fatty acid patterns of phospholipids and lipopolysaccharides in the characterisation of microbial communities in soil: a review," Biology and Fertility of Soils, vol. 29, no. 2, pp. 111-129, 1999.

[9] J. Six, S. D. Frey, R. K. Thiet, and K. M. Batten, "Bacterial and fungal contributions to carbon sequestration in agroecosystems," Soil Science Society of America Journal, vol. 70, no. 2, pp. 555-569, 2006.

[10] K. Schreiner, N. Blair, W. Levinson, and L. Egerton-Warburton, "Contribution of fungal macromolecules to soil carbon sequestration," in Soil Carbon, E. Hartemink and K. McSweeney, Eds., Progress in Soil Science, pp. 155-161, Springer International, 2014.

[11] F. J. Stevenson, "Organic forms of soil nitrogen," in Nitrogen in Agricultural Soils, American Society of Agronomy, Madison, Wis, USA, 1982.
[12] A. Ekblad and T. Nasholm, "Determination of chitin in fungi and mycorrhizal roots by an improved HPLC analysis of glucosamine," Plant and Soil, vol. 178, no. 1, pp. 29-35, 1996.

[13] M. J. Swift, “The estimation of mycelial biomass by determination of the hexosamine content of wood tissue decayed by fungi," Soil Biology and Biochemistry, vol. 5, no. 3, pp. 321-332, 1973.

[14] J. P. Ride and R. B. Drysdale, "A rapid method for the chemical estimation of filamentous fungi in plant tissue," Physiological Plant Pathology, vol. 2, no. 1, pp. 7-15, 1972.

[15] J. Parsons, "Chemistry and distribution of amino sugars in soils and soil organisms," in Soil Biochemistry, Marcel Dekker, New York, NY, USA, 1981.

[16] M. T. Madigan, Brock Biology of Microorganisms, SciELO Espana, 11th edition, 2005.

[17] B. Engelking, H. Flessa, and R. G. Joergensen, "Shifts in amino sugar and ergosterol contents after addition of sucrose and cellulose to soil," Soil Biology and Biochemistry, vol. 39, no. 8, pp. 2111-2118, 2007.

[18] L. A. Elson and W. T. J. Morgan, "A colorimetric method for the determination of glucosamine and chondrosamine," Biochemical Journal, vol. 27, no. 6, pp. 1824-1828, 1933.

[19] W. T. J. Morgan and L. A. Elson, "A colorimetric method for the determination of $\mathrm{N}$-acetylglucosamine and $\mathrm{N}$-acetylchrondrosamine," Biochemical Journal, vol. 28, no. 3, pp. 988-995, 1934.

[20] A. Tsuji, T. Kinoshita, and M. Hoshino, "Analytical chemical studies on amino sugars. II. Determination of hexosamines using 3-methyl-2-benzothiazolone hydrazone hydrochloride," Chemical \& Pharmaceutical Bulletin, vol. 17, no. 7, pp. 1505-1510, 1969.

[21] A. Tsuji, T. Kinoshita, and M. Hoshino, "Microdetermination of hexosamines," Chemical and Pharmaceutical Bulletin, vol. 17, no. 1, pp. 217-218, 1969.

[22] W. Amelung, "Methods using amino sugars as markers for microbial residues in soil," in Assessment Methods for Soil Carbon, pp. 233-272, CRC/Lewis Publishers, Boca Raton, Fla, USA, 2001.

[23] C. Liang, J. A. Pedersen, and T. C. Balser, "Aminoglycoside antibiotics may interfere with microbial amino sugar analysis," Journal of Chromatography A, vol. 1216, no. 27, pp. 5296-5301, 2009.

[24] X. Zhang and W. Amelung, "Gas chromatographlc determination of muramic acid, glucosamine, mannosamine, and galactosamine in soils," Soil Biology and Biochemistry, vol. 28, no. 9, pp. 1201-1206, 1996.

[25] J. Díaz, J. L. Lliberia, L. Comellas, and F. Broto-Puig, "Amino acid and amino sugar determination by derivatization with 6-aminoquinolyl- $N$-hydroxysuccinimidyl carbamate followed by high-performance liquid chromatography and fluorescence detection," Journal of Chromatography A, vol. 719, no. 1, pp. 171179, 1996.

[26] A. Appuhn, R. G. Joergensen, M. Raubuch, E. Scheller, and B. Wilke, "The automated determination of glucosamine, galactosamine, muramic acid, and mannosamine in soil and root hydrolysates by HPLC," Journal of Plant Nutrition and Soil Science, vol. 167, no. 1, pp. 17-21, 2004.

[27] L. Zelles, "The simultaneous determination of muramic acid and glucosamine in soil by high-performance liquid chromatography with precolumn fluorescence derivatization," Biology and Fertility of Soils, vol. 6, no. 2, pp. 125-130, 1988. 
[28] K. Kaiser and R. Benner, "Determination of amino sugars in environmental samples with high salt content by high-performance anion-exchange chromatography and pulsed amperometric detection," Analytical Chemistry, vol. 72, no. 11, pp. 2566-2572, 2000.

[29] S. Bodé, K. Denef, and P. Boeckx, "Development and evaluation of a high-performance liquid chromatography/isotope ratio mass spectrometry methodology for $\delta 13 \mathrm{C}$ analyses of amino sugars in soil," Rapid Communications in Mass Spectrometry, vol. 23, no. 16, pp. 2519-2526, 2009.

[30] C. Indorf, F. Stamm, J. Dyckmans, and R. G. Joergensen, "Determination of saprotrophic fungi turnover in different substrates by glucosamine-specific $\delta 13 \mathrm{C}$ liquid chromatography/isotope ratio mass spectrometry," Fungal Ecology, vol. 5, no. 6, pp. 694701, 2012.

[31] C. Decock, K. Denef, S. Bodé, J. Six, and P. Boeckx, "Critical assessment of the applicability of gas chromatography-combustion-isotope ratio mass spectrometry to determine amino sugar dynamics in soil," Rapid Communications in Mass Spectrometry, vol. 23, no. 8, pp. 1201-1211, 2009.

[32] A. Roda, L. Sabatini, A. Barbieri et al., "Development and validation of a sensitive HPLC-ESI-MS/MS method for the direct determination of glucosamine in human plasma," Journal of Chromatography B, vol. 844, no. 1, pp. 119-126, 2006.

[33] S. Zhong, D. Zhong, and X. Chen, "Improved and simplified liquid chromatography/electrospray ionization mass spectrometry method for the analysis of underivatized glucosamine in human plasma," Journal of Chromatography B, vol. 854, no. 1-2, pp. 291-298, 2007.

[34] E. Pastorini, R. Rotini, M. Guardigli et al., "Development and validation of a HPLC-ES-MS/MS method for the determination of glucosamine in human synovial fluid," Journal of Pharmaceutical and Biomedical Analysis, vol. 50, no. 5, pp. 1009-1014, 2009.

[35] B. Dejaegher, D. Mangelings, and Y. Vander Heyden, "Method development for HILIC assays," Journal of Separation Science, vol. 31, no. 9, pp. 1438-1448, 2008.

[36] W. Amelung, X. Zhang, K. W. Flach, and W. Zech, "Amino sugars in native grassland soils along a climosequence in North America," Soil Science Society of America Journal, vol. 63, no. 1, pp. 86-92, 1999.

[37] G. E. Black, A. Fox, K. Fox, A. P. Snyder, and P. B. W. Smith, "Electrospray tandem mass spectrometry for analysis of native muramic acid in whole bacterial cell hydrolyzates," Analytical Chemistry, vol. 66, no. 23, pp. 4171-4176, 1994. 

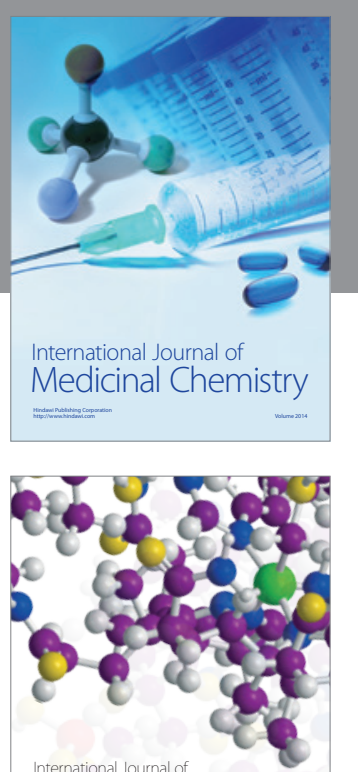

Carbohydrate Chemistry

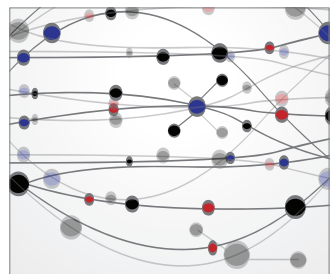

The Scientific World Journal
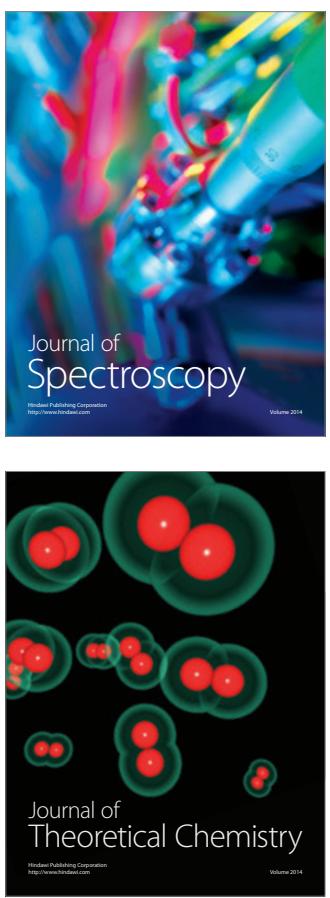
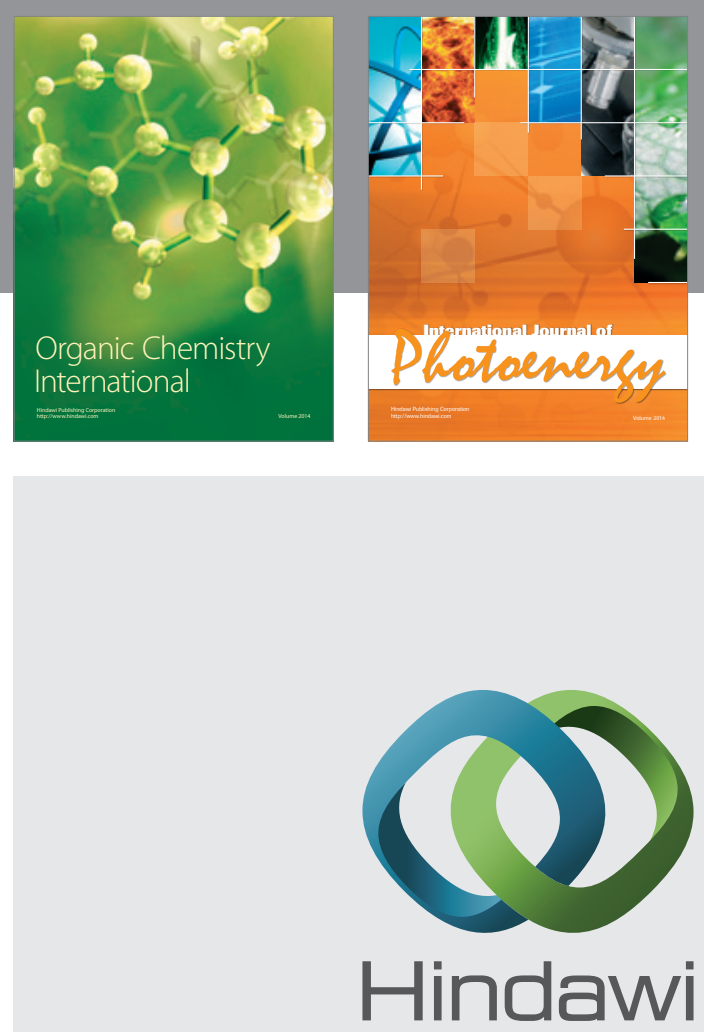

Submit your manuscripts at

http://www.hindawi.com

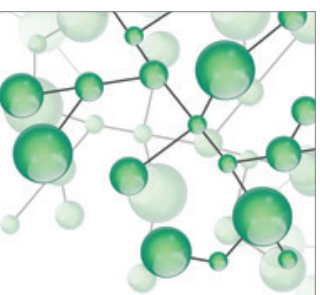

International Journal of

Inorganic Chemistry

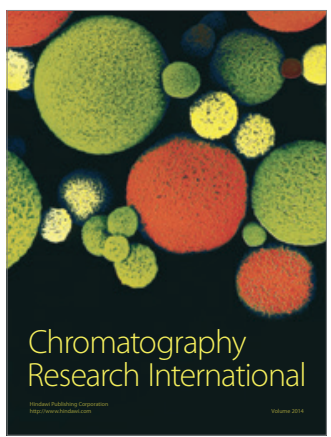

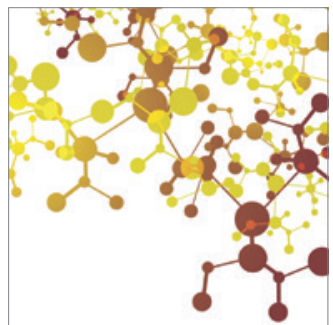

Applied Chemistry
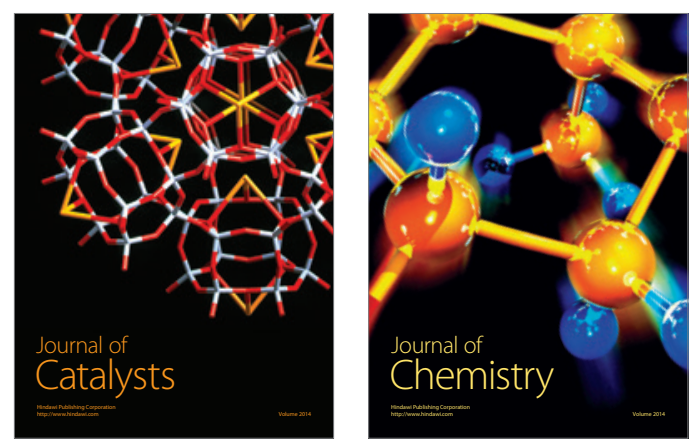
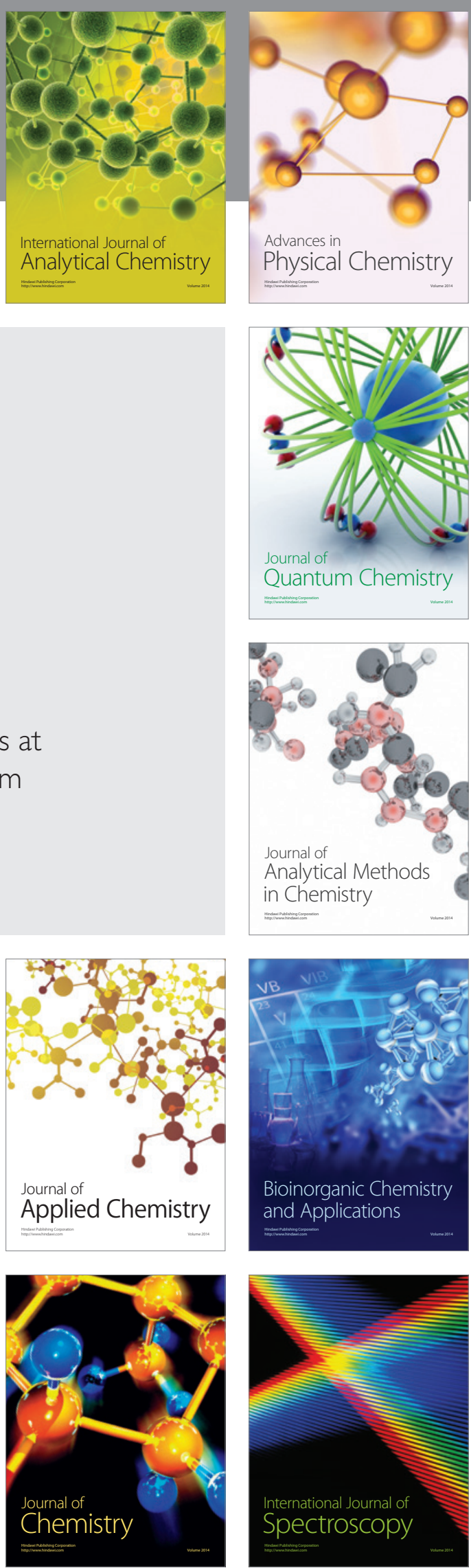